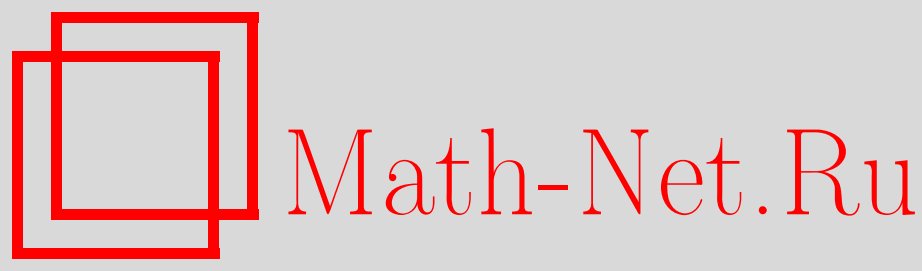

С. Н. Лакаев, Ш. М. Латипов, О существовании и аналитичности собственных значений двухканальной молекулярно-резонансной модели, ТМФ, 2011, том 169, номер 3, 341-351

DOI: https://doi.org/10.4213/tmf6734

Использование Общероссийского математического портала Math-Net.Ru подразумевает, что вы прочитали и согласны с пользовательским соглашением http: //www . mathnet.ru/rus/agreement

Параметры загрузки:

IP : 3.85 .7 .115

26 апреля 2023 г., $17: 02: 42$

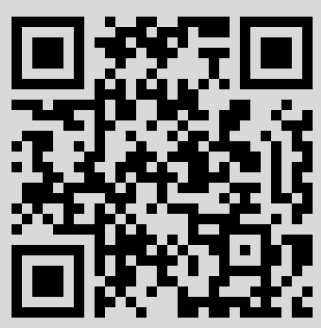




\title{
ФИЗИКА
}

Том 169, № 3

декабрь, 2011

(C) 2011 г. $\quad$ С. Н. Лакаев*†, Ш. М. Латипов*

\section{О СУЩЕСТВОВАНИИ И АНАЛИТИЧНОСТИ СОБСТВЕННЫХ ЗНАЧЕНИЙ ДВУХКАНАЛЬНОЙ МОЛЕКУЛЯРНО-РЕЗОНАНСНОЙ МОДЕЛИ}

\begin{abstract}
Рассмотрено семейство операторов $H_{\gamma \mu}(k), k \in \mathbb{T}^{d}:=(-\pi, \pi]^{d}$, ассоциированное гамильтонианом системы, состоящей из не более чем двух частиц на $d$-мерной решетке $\mathbb{Z}^{d}$, взаимодействующих как с помощью парного контактного потенциала $(\mu>0)$, так и с помощью операторов рождения и уничтожения $(\gamma>0)$. Доказано существование единственного собственного значения оператора $H_{\gamma \mu}(k), k \in \mathbb{T}^{d}$, или его отсутствие в зависимости как от параметров взаимодействий $\gamma, \mu \geqslant 0$, так и от квазиимпульса системы $k \in \mathbb{T}^{d}$. Показана аналитичность соответствующего собственного вектора. Установлено, что собственное значение и собственный вектор являются аналитическими функциями квазиимпульса $k \in \mathbb{T}^{d}$ в области существования $G \subset \mathbb{T}^{d}$.
\end{abstract}

Ключевые слова: гамильтониан, оператор рождения, собственные значения, связанное состояние, решетка.

\section{1. ПОСТАНОВКА ЗАДАЧИ}

Пусть $\mathcal{H}=\mathcal{H}_{0} \oplus \mathcal{H}_{1}$ - двухканальное гильбертово пространство, состоящее из одномерного гильбертова пространства $\mathcal{H}_{0}=C^{1}$ (канал 1) и ядерного гильбертова пространства $\mathcal{H}_{1}=L_{e}^{2}\left(\mathbb{T}^{d}\right)$ - четных, квадратично-интегрируемых функций на $d$-мерном $(d \geqslant 1)$ торе $\mathbb{T}^{d}$ (канал 2$)$. Элементы пространства $\mathcal{H}$ представляются как векторы $u=\left(f_{0}, f_{1}\right)$, где $f_{0} \in \mathcal{H}_{0}, f_{1} \in \mathcal{H}_{1}$. Скалярное произведение

$$
\langle f, g\rangle=\left(f_{0}, g_{0}\right)+\left(f_{1}, g_{1}\right)
$$

в $\mathcal{H}$ естественно определяется через скалярные произведения

$$
\left(f_{0}, g_{0}\right)_{\mathcal{H}_{0}}=f_{0} \cdot \bar{g}_{0}, \quad\left(f_{1}, g_{1}\right)_{\mathcal{H}_{1}}=\int_{\mathbb{T}^{d}} f_{1}(t) \bar{g}_{1}(t) d t
$$

Пусть $E(k), k=\left(k^{(1)}, \ldots, k^{(d)}\right) \in \mathbb{T}^{d},-$ скалярный оператор, действующий в гильбертовом пространстве $\mathcal{H}_{0}$ по формуле

$$
E(k) f_{0}=\varepsilon(k) f_{0}, \quad f_{0} \in \mathcal{H}_{0},
$$

*Самаркандский государственный университет, Самарканд, Узбекистан.

E-mail: sherdorlatipov@mail.ru

${ }^{\dagger}$ Самаркандское отделение АН РУз, Самарканд, Узбекистан. E-mail: slakaev@mail.ru 
где $\varepsilon(k)=\sum_{i=1}^{d}\left(1-\cos k^{(i)}\right)$, а $H_{\mu}(k), k \in \mathbb{T}^{d},-$ двухчастичный дискретный оператор Шредингера, ассоциированный гамильтонианом системы двух одинаковых частиц (бозонов), взаимодействующих с помощью парного контактного потенциала $\mu \geqslant 0$.

Оператор $H_{\mu}(k), k \in \mathbb{T}^{d}$, действует в гильбертовом пространстве $\mathcal{H}_{1}$ по формуле

$$
H_{\mu}(k)=H_{0}(k)-\mu V,
$$

где $H_{0}(k)$ - оператор умножения на функцию $\varepsilon_{k}(\cdot)$ :

$$
\begin{gathered}
\left(H_{0}(k) f_{1}\right)(q)=\varepsilon_{k}(q) f_{1}(q), \\
\varepsilon_{k}(q)=\varepsilon\left(\frac{k}{2}-q\right)+\varepsilon\left(\frac{k}{2}+q\right)=2 \sum_{i=1}^{d}\left(1-\cos \frac{k^{(i)}}{2} \cos q^{(i)}\right), \quad f_{1} \in \mathcal{H}_{1},
\end{gathered}
$$

и $V$ - неотрицательный интегральный оператор ранга один:

$$
\left(V f_{1}\right)(q)=\alpha_{0}\left(f_{1}, \alpha_{0}\right)_{\mathcal{H}_{1}}=\frac{1}{(2 \pi)^{d}} \int_{\mathbb{T}^{d}} f_{1}(s) d s, \quad \alpha_{0} \equiv(2 \pi)^{-d / 2}, \quad f_{1} \in \mathcal{H}_{1} .
$$

Рассмотрим семейство операторов $H_{\gamma \mu}(k), k \in \mathbb{T}^{d}$, ассоциированное гамильтонианом системы, состоящей из не более чем двух частиц на $d$-мерной решетке $\mathbb{Z}^{d}$, взаимодействующих как с помощью парного контактного потенциала $(\mu>0)$, так и с помощью операторов рождения и уничтожения $(\gamma>0)$.

Оператор $H_{\gamma \mu}(k), k \in \mathbb{T}^{d}, \gamma, \mu \in[0,+\infty)$, действует в гильбертовом пространстве $\mathcal{H}$ по формуле

$$
H_{\gamma \mu}(k)\left(\begin{array}{c}
f_{0} \\
f_{1}(q)
\end{array}\right)=\left(\begin{array}{c}
E(k) f_{0}+C_{\gamma}^{*} f_{1} \\
C_{\gamma} f_{0}+\left(H_{\mu}(k) f_{1}\right)(q)
\end{array}\right),
$$

где $C_{\gamma}^{*} f_{1}=\gamma\left(f_{1}, \alpha_{0}\right) \mathcal{H}_{1}$ и $C_{\gamma} f_{0}=\gamma\left(f_{0}, \alpha_{0}\right) \mathcal{H}_{0}$ - операторы рождения и уничтожения соответственно. Легко проверить, что $H_{\gamma \mu}(k), k \in \mathbb{T}^{d}, \gamma, \mu \in[0,+\infty)$, является ограниченным и самосопряженным оператором в $\mathcal{H}$.

В работе [1] была рассмотрена аналогичная, не зависящая от параметра $k \in \mathbb{T}^{d}$, молекулярно-резонансная модель и изучены ее собственные значения и резонансы. В работе [2] рассмотрен гамильтониан системы, состоящей из не более чем трех частиц, и показано существование виртуальных уровней у модельного оператора $H_{\gamma 0}(k)$, что влечет наличие бесконечного числа связанных состояний (собственных значений) гамильтониана.

В работе [3] для широкого класса двухчастичных дискретных операторов Шредингера $H(k), k \in \mathbb{T}^{d}, d \geqslant 3$, ассоциированных гамильтонианом системы двух частиц, взаимодействующих с помощью парных короткодействующих потенциалов, показано, что если неотрицательный оператор $H(0)$ имеет виртуальный уровень или собственное значение на дне существенного спектра, то при всех ненулевых значениях $k \in \mathbb{T}^{d}, d \geqslant 3$, оператор $H(k), k \in \mathbb{T}^{d}$, имеет собственное значение, лежащее левее существенного спектра. Двухчастичный дискретный оператор Шредингера $H(k)$, $k \in \mathbb{T}^{d}$, характеризуется еще и тем, что если $H(0)$ имеет собственное значение, то $H(k)$ также имеет собственное значение при всех ненулевых значениях квазиимпульса $k \in \mathbb{T}^{d}$ (см. теорему 5.4 в работе [3]).

В настоящей работе доказано существование единственного собственного значения оператора $H_{\gamma \mu}(k), k \in \mathbb{T}^{d}$, или его отсутствие в зависимости как от параметров 
взаимодействий $\gamma, \mu \geqslant 0$, так и от квазиимпульса системы $k \in \mathbb{T}^{d}$. Показана аналитичность соответствующего собственного вектора. Кроме того, доказано, что собственное значение и соответствующий собственный вектор являются аналитическими функциями квазиимпульса $k \in \mathbb{T}^{d}$ в области существования $G \subset \mathbb{T}^{d}$. В частности, в теореме 4 при $d \geqslant 3$ установлено, что оператор $H_{\gamma \mu}(k), k \in \mathbb{T}^{d}$, имеет собственное значение в некоторой окрестности множества векторов $k=\left(k^{(1)}, \ldots, k^{(d)}\right)$, у которых каждая координата равна либо нулю, либо $\pi$, и не имеет собственных значений при других значениях квазиимпульса $k \in \mathbb{T}^{d}$.

Этот результат отличает рассматриваемую модель $H_{\gamma \mu}(k), k \in \mathbb{T}^{d}$, от двухчастичного дискретного оператора Шредингера $H(k), k \in \mathbb{T}^{d}$, и является следствием наличия взаимодействий с помощью операторов рождения и уничтожения.

\section{2. ФОРМУЛИРОВКА ОСНОВНЫХ РЕЗУЛЬТАТОВ}

Так как разность операторов $H_{\gamma \mu}(k)$ и $H_{0 \mu}(k), k \in \mathbb{T}^{d}$, является оператором ранга два, то в силу известной теоремы Вейля [4] существенный спектр оператора $H_{\gamma \mu}(k)$, $k \in \mathbb{T}^{d}$, совпадает с существенным спектром $\sigma_{\text {ess }}\left(H_{0 \mu}(k)\right)$ оператора $H_{0 \mu}(k), k \in \mathbb{T}^{d}$, а именно верны равенства

$$
\sigma_{\mathrm{ess}}\left(H_{\gamma \mu}(k)\right)=\sigma_{\mathrm{ess}}\left(H_{0 \mu}(k)\right)=\sigma_{\mathrm{ess}}\left(h_{\mu}(k)\right)=\sigma\left(h_{0}(k)\right)=\left[\varepsilon_{\min }(k), \varepsilon_{\max }(k)\right],
$$

где

$\varepsilon_{\min }(k)=\min _{q \in \mathbb{T}^{d}} \varepsilon_{k}(q)=2 \sum_{i=1}^{d}\left(1-\cos \frac{k^{(i)}}{2}\right), \quad \varepsilon_{\max }(k)=\max _{q \in \mathbb{T}^{d}} \varepsilon_{k}(q)=2 \sum_{i=1}^{d}\left(1+\cos \frac{k^{(i)}}{2}\right)$.

Одним из основных результатов работы является следующая теорема, утверждающая существование и аналитичность единственного собственного значения и соответствующего собственного вектора оператора $H_{\gamma \mu}(k), k \in \mathbb{T}^{d}$, при $d=1,2$.

Теорема 1. Пусть $d=1,2 u \gamma \geqslant 0, \mu \geqslant 0, \gamma+\mu>0$. Тогда для любого $k \in$ $\mathbb{T}^{d}$ оператор $H_{\gamma \mu}(k)$ имеет единственное собственное значение $E_{\gamma \mu}(k)<\varepsilon_{\min }(k)$. Соответствующий собственный вектор $f_{k}=\left(f_{0, k}, f_{1, k}\right) \in \mathcal{H}$ имеет вид

$$
f_{0, k}=\frac{-c \gamma}{\varepsilon(k)-E_{\gamma \mu}(k)}, \quad f_{1, k}(q)=\left(\frac{\gamma^{2}}{\varepsilon(k)-E_{\gamma \mu}(k)}+\mu\right) \frac{c}{\varepsilon_{k}(q)-E_{\gamma \mu}(k)}
$$

и является аналитическим на $\mathbb{T}^{d}$, где $c \neq 0$ - нормирующий множитель.

Более того, отображение $E_{\gamma \mu}: \mathbb{T}^{d} \rightarrow \mathbb{R}, k \rightarrow E_{\gamma \mu}(k)$ является четной и вещественно-аналитической функцией на $\mathbb{T}^{d}$, а отображсение $f: \mathbb{T}^{d} \rightarrow \mathcal{H}, k \rightarrow f_{k} \in \mathcal{H}$ векторнозначным аналитическим отображением на $\mathbb{T}^{d}$.

Пусть $0 \leqslant n \leqslant d$. Для любого фиксированного $0 \leqslant \chi \leqslant \pi$ определим следующие множества:

$$
\begin{gathered}
\Pi_{n, \chi}=\left\{k=\left(k^{(1)}, \ldots, k^{(d)}\right) \in \mathbb{T}^{d}: \text { ровно } n \text { координат у вектора } k \text { равны } \chi\right\}, \\
\Pi_{\chi}^{(3)}=\bigcup_{n=0}^{d-3} \Pi_{n, \chi}, \quad \widetilde{\Pi}_{\xi, \eta}=\bigcup_{i=0}^{d}\left(\Pi_{i, \xi} \cap \Pi_{d-i, \eta}\right), \quad \xi \neq \eta .
\end{gathered}
$$


Таким образом, $\Pi_{\chi}^{(3)}$ - множество векторов $k=\left(k^{(1)}, \ldots, k^{(d)}\right) \in \mathbb{T}^{d}$, у которых по крайней мере три координаты отличны от $\chi$, и $\widetilde{\Pi}_{\xi, \eta}-$ множество векторов $k=$ $\left(k^{(1)}, \ldots, k^{(d)}\right) \in \mathbb{T}^{d}$, у которых все координаты равны либо $\xi$, либо $\eta$. Обозначим через $\widetilde{\Pi}_{\xi, \eta}^{\perp}$ множество векторов $k=\left(k^{(1)}, \ldots, k^{(d)}\right) \in \mathbb{T}^{d}$, у которых хотя бы одна координата отлична от $\xi$ и $\eta$, т.е. $\widetilde{\Pi}_{\xi, \eta}^{\perp}=\mathbb{T}^{d} \backslash \widetilde{\Pi}_{\xi, \eta}$.

Следующая теорема утверждает наличие собственных значений оператора $H_{\gamma \mu}(k)$ в случае $d \geqslant 3$ и $\varepsilon_{\min }(k)=\varepsilon(k)$, т.е. при $k \in \widetilde{\Pi}_{0, \pi}$.

ТЕорема 2. При любъх $\gamma>0, \mu \geqslant 0 u k \in \widetilde{\Pi}_{0, \pi}$ оператор $H_{\gamma \mu}(k)$ имеет единственное собственное значение, лежащее левее $\varepsilon_{\min }(k)$.

ЗАмечАниЕ 1 . Пусть $L_{e}^{1}\left(\mathbb{T}^{d}\right)$ - банахово пространство четных интегрируемых функций на $\mathbb{T}^{d}$. Заметим, что если для некоторого $k \in \Pi_{\pi}^{(3)} \cap \widetilde{\Pi}_{0, \pi}^{\perp}$ уравнение $H_{\gamma \mu}(k) f_{k}=\varepsilon_{\min }(k) f_{k}$ имеет ненулевое решение $f_{k}=\left(f_{0, k}, f_{1, k}\right)$, то для его координат $f_{0, k}$ и $f_{1, k}$ выполняются следующие соотношения:

1) $f_{0, k} \in \mathcal{H}_{0}, f_{1, k}(q) \in L_{e}^{1}\left(\mathbb{T}^{d}\right) \backslash L_{e}^{2}\left(\mathbb{T}^{d}\right)$ при $k \in\left\{\Pi_{d-3, \pi} \cup \Pi_{d-4, \pi}\right\} \cap \widetilde{\Pi}_{0, \pi}^{\perp}$, где $\Pi_{-1, \pi}=\varnothing$;

2) $f_{0, k} \in \mathcal{H}_{0}, f_{1, k}(q) \in L_{e}^{2}\left(\mathbb{T}^{d}\right)$ при $k \in \Pi_{\pi}^{(3)} \cap \widetilde{\Pi}_{0, \pi}^{\perp}$.

ОПРЕДЕЛЕНИЕ. Если выполняются соотношения из п. 1 замечания 1, говорят, что дно (левый край) существенного спектра $\varepsilon_{\min }(k)$ является виртуальным уровнем оператора $H_{\gamma \mu}(k)$.

Следующая теорема утверждает, что левый край существенного спектра оператора $H_{\gamma \mu}(k)$ не является ни виртуальным уровнем, ни собственным значением в случае $\varepsilon(k)=\varepsilon_{\min }(k)$, т.е. при $k \in \widetilde{\Pi}_{0, \pi}$.

Теорема 3. Пусть $d \geqslant 1, \gamma>0 u \mu \geqslant 0$. Тогда справедливы следующие утверждения.

А. Если $k \in \widetilde{\Pi}_{0, \pi} \backslash\{\vec{\pi}\}$, где $\vec{\pi}=(\pi, \ldots, \pi)$, т.е. каждая из координат вектоpa $k$ равна либо нулю, либо $\pi$, то число $z=\varepsilon_{\min }(k)$ не является ни собственным значением, ни виртуальным уровнем оператора $H_{\gamma \mu}(k)$.

Б. Если $k=\vec{\pi}=(\pi, \ldots, \pi)$, то число $z=\varepsilon_{\min }(k)$ является собственным значением оператора $H_{\gamma \mu}(k)$ бесконечной кратности. Более того, все соответствующие собственные векторы ортогональны единичному вектору $1 \in \mathcal{H}_{1}$.

Обозначим через $a(k, z)$ вещественно-аналитическую в $\mathbb{T}^{d} \times\left(\mathbb{C} \backslash \sigma_{\mathrm{ess}}\left(H_{\gamma \mu}(k)\right)\right)$ функцию:

$$
a(k, z):=\left(\alpha_{0}, r_{0}(k, z) \alpha_{0}\right)=\frac{1}{(2 \pi)^{d}} \int_{\mathbb{T}^{d}} \frac{d q}{\varepsilon_{k}(q)-z} .
$$

Для каждого фиксированного $k \in \mathbb{T}^{d}$ определим аналитическую в $\mathbb{C} \backslash\left[\varepsilon_{\min }(k), \varepsilon_{\max }(k)\right]$ функцию (определитель Фредгольма)

$$
\Delta_{\gamma \mu}(k ; z)=1-\left(\frac{\gamma^{2}}{\varepsilon(k)-z}+\mu\right) a(k, z) .
$$

Пусть $d \geqslant 3$. Тогда для любого $k \in \Pi_{\pi}^{(3)}$ существует конечный предел (см. ниже лемму 4)

$$
a\left(k, \varepsilon_{\min }(k)\right)=\lim _{z \rightarrow \varepsilon_{\min }(k)-0} a(k ; z)>0,
$$


и, следовательно, для любого $k \in \Pi_{\pi}^{(3)} \cap \widetilde{\Pi}_{0, \pi}^{\perp}$ существует конечный предел

$$
\Delta_{\gamma \mu}(k)=\lim _{z \rightarrow \varepsilon_{\min }(k)-0} \Delta_{\gamma \mu}(k ; z)=\Delta_{\gamma \mu}\left(k ; \varepsilon_{\min }(k)\right) .
$$

Отметим, что $\Delta_{\gamma \mu}(\cdot)$ является вещественно-аналитической функцией на $\Pi_{\pi}^{(3)} \cap \widetilde{\Pi}_{0, \pi}^{\perp} \subset$ $\mathbb{T}^{d}$ (см. утверждение А леммы 4). Для любого $k \in \Pi_{\pi}^{(3)} \cap \widetilde{\Pi}_{0, \pi}^{\perp}$ определим $\Delta_{\gamma \mu}^{*}(k)$ следующим образом:

$$
\Delta_{\gamma \mu}^{*}(k)=\varepsilon(k)-\varepsilon_{\min }(k)-\left[\gamma^{2}+\mu\left(\varepsilon(k)-\varepsilon_{\min }(k)\right)\right] a\left(k, \varepsilon_{\min }(k)\right),
$$

где

$$
\varepsilon(k)-\varepsilon_{\min }(k)=\sum_{i=1}^{d} 2 \cos \frac{k_{i}}{2}\left(1-\cos \frac{k_{i}}{2}\right) .
$$

Лемма 1. Пусть $d \geqslant 3, k \in \Pi_{\pi}^{(3)} \cap \widetilde{\Pi}_{0, \pi}^{\perp}$. Число $z=\varepsilon_{\min }(k)$ является виртуальным уровнем при $k \in\left\{\Pi_{d-3, \pi} \cup \Pi_{d-4, \pi}\right\} \cap \widetilde{\Pi}_{0, \pi}^{\perp}$ и собственным значением при $k \in \Pi_{\pi}^{(3)} \cap \widetilde{\Pi}_{0, \pi}^{\perp}$ оператора $H_{\gamma \mu}(k)$ тогда и только тогда, когда $\Delta_{\gamma \mu}^{*}(k)=0$.

Для любых $\gamma, \mu, \gamma+\mu>0$ имеют место соотношения

$$
\begin{aligned}
& \Delta_{\min }(\gamma, \mu)=\inf _{k \in \Pi_{\pi}^{(3)}} \Delta_{\gamma \mu}(k)=-\infty, \\
& \Delta_{\max }(\gamma, \mu)=\max _{k \in \Pi_{\pi}^{(3)}} \Delta_{\gamma \mu}(k)<+\infty .
\end{aligned}
$$

Введем следующие множества:

$$
\begin{aligned}
& G_{\gamma \mu}^{0}=\left\{k \in \Pi_{\pi}^{(3)} \cap \widetilde{\Pi}_{0, \pi}^{\perp}: \Delta_{\gamma \mu}(k)=0\right\}, \\
& G_{\gamma \mu}^{<}=\left\{k \in \Pi_{\pi}^{(3)} \cap \widetilde{\Pi}_{0, \pi}^{\perp}: \Delta_{\gamma \mu}(k)<0\right\}, \\
& G_{\gamma \mu}^{>}=\left\{k \in \Pi_{\pi}^{(3)} \cap \widetilde{\Pi}_{0, \pi}^{\perp}: \Delta_{\gamma \mu}(k)>0\right\} .
\end{aligned}
$$

Заметим, что непустое множество $G_{\gamma \mu}^{0} \subset \Pi_{\pi}^{(3)} \cap \widetilde{\Pi}_{0, \pi}^{\perp}$ является поверхностью (многообразием) коразмерности 1 , а $G_{\gamma \mu}^{<} \subset \Pi_{\pi}^{(3)} \cap \widetilde{\Pi}_{0, \pi}^{\perp}$ и $G_{\gamma \mu}^{>} \subset \Pi_{\pi}^{(3)} \cap \widetilde{\Pi}_{0, \pi}^{\perp}$ - открытыми связными множествами в $\mathbb{T}^{d}$.

Следующая теорема утверждает наличие или отсутствие собственных значений и виртуальных уровней оператора $H_{\gamma \mu}(k)$ в случае $\varepsilon(k)<\varepsilon_{\min }(k)$, т.е. при $k \in \widetilde{\Pi}_{0, \pi}^{\perp}$.

ТеОрема 4. Предположим, что $d \geqslant 3$. Справедливы следующие утверждения.

A. Пусть $\gamma, \mu \geqslant 0, \gamma+\mu>0, k \in \Pi_{\pi}^{(3)} \cap \widetilde{\Pi}_{0, \pi}^{\perp} u \Delta_{\max }(\gamma, \mu)<0$, тогда $G_{\gamma \mu}^{<}=$ $\Pi_{\pi}^{(3)} \cap \widetilde{\Pi}_{0, \pi}^{\perp}$.

Б. Для любого $k \in G_{\gamma \mu}^{<}$оператор $H_{\gamma \mu}(k)$ имеет единственное собственное значение $E_{\gamma \mu}(k)<\varepsilon_{\min }(k)$. Соответствующий собственный вектор $f_{k}=\left(f_{0, k}, f_{1, k}\right) \in \mathcal{H}$ имеет вид

$$
f_{0, k}=\frac{-c \gamma}{\varepsilon(k)-E_{\gamma \mu}(k)}, \quad f_{1, k}(q)=\left(\frac{\gamma^{2}}{\varepsilon(k)-E_{\gamma \mu}(k)}+\mu\right) \frac{c}{\varepsilon_{k}(q)-E_{\gamma \mu}(k)}
$$

и является аналитическим в $\mathbb{T}^{d}$, где $c \neq 0$ - нормирующий множитель. При этом функиия $E_{\gamma \mu}(\cdot)$ является четной аналитической функцией в $G_{\gamma \mu}^{<}$. Отображение 
$f: G_{\gamma \mu}^{<} \longrightarrow \mathcal{H}, k \longrightarrow f_{k} \in \mathcal{H}$ является векторнозначным аналитическим отображением.

В. Пусть $\gamma, \mu \geqslant 0, \gamma+\mu>0 u k \in G_{\gamma \mu}^{0}$. Тогда число $z=\varepsilon_{\min }(k)$ является виртуальным уровнем при $k \in\left[\Pi_{d-3, \pi} \cup \Pi_{d-4, \pi}\right] \cap \widetilde{\Pi}_{0, \pi}^{\perp}$ (собственным значением при $\left.k \in \Pi_{\pi}^{(3)} \cap \widetilde{\Pi}_{0, \pi}^{\perp}\right)$ оператора $H_{\gamma \mu}(k)$. Соответствующее виртуальное (связанное) состояние $f_{k}$ имеет вид $f_{k}=\left(f_{0, k}, f_{1, k}\right)$,

$$
f_{0, k}=\frac{-c \gamma}{\varepsilon(k)-\varepsilon_{\min }(k)}, \quad f_{1, k}(q)=\left(\frac{\gamma^{2}}{\varepsilon(k)-\varepsilon_{\min }(k)}+\mu\right) \frac{c}{\varepsilon_{k}(q)-\varepsilon_{\min }(k)},
$$

где $c \neq 0$ - нормирующий множитель.

Г. Пусть $\gamma>0, \mu \geqslant 0 u k \in G_{\gamma \mu}^{0} \cup G_{\gamma \mu}^{>}$. Тогда оператор $H_{\gamma \mu}(k)$ не имеет собственного значения, лежащего левее точки $\varepsilon_{\min }(k)$.

СледСТВиЕ. Пусть $\gamma=0$. Для любого $k \in \Pi_{\pi}^{(3)}$ определим положительное число

$$
\mu_{0}(k)=\left(\frac{1}{(2 \pi)^{d}} \int_{\mathbb{T}^{d}} \frac{d q}{\varepsilon_{k}(q)-\varepsilon_{\min }(k)}\right)^{-1} .
$$

Тогда при всех $\mu>\mu_{0}(0)$ условие утверждения А теоремы 4 выполняется, и, следовательно, оператор $H_{0 \mu}(k)$ имеет единственное собственное значение $E_{0 \mu}(k)<$ $\varepsilon_{\min }(k)$.

ЗАмЕчАниЕ 2. Заметим, что утверждение А теоремы 4 выполняется при достаточно больших $\gamma, \mu>0$, а утверждение $\Gamma$ теоремы 4 выполняется при достаточно малых $\gamma, \mu \geqslant 0, \mu+\gamma>0$. Таким образом, при малых взаимодействиях $\gamma, \mu \geqslant 0$, $\mu+\gamma>0$, оператор $H_{\gamma \mu}(k), k \in \Pi_{\pi}^{(3)} \cap \widetilde{\Pi}_{0, \pi}^{\perp}$, не имеет собственного значения, лежащего левее существенного спектра.

\section{3. ДОКАЗАТЕЛЬСТВО ОСНОВНЫХ РЕЗУЛЬТАТОВ}

Лемма 2. А. Для любого $k \in \mathbb{T}^{d}$ функиия $a(k, \cdot)$ положительна и монотонно возрастает на интервале $\left(-\infty, \varepsilon_{\min }(k)\right)$ и для любого $z \in \mathbb{C} \backslash\left[\varepsilon_{\min }(k), \varepsilon_{\max }(k)\right]$ она вещественно-аналитична на $\mathbb{T}^{d}$.

Б. Для любых $\gamma, \mu \in[0,+\infty), k \in \mathbb{T}^{d}$ функиия (4) монотонно убывает на интервале $\left(-\infty, \varepsilon_{\min }(k)\right)$.

В. Для любъх $\gamma, \mu \in[0,+\infty), k \in \mathbb{T}^{d}$ нули функций $\Delta_{\gamma \mu}^{*}(k ; \cdot) u \Delta_{\gamma \mu}(k ; \cdot)$ совпадают на $\left(-\infty, \varepsilon_{\min }(k)\right)$, и функция $\Delta_{\gamma \mu}^{*}(k ; \cdot)$ на интервале $\left(-\infty, \varepsilon_{\min }(k)\right)$ имеет не более одного нуля.

ДоказАтельство. Утверждения А, Б леммы очевидны. Докажем утверждение В. При всех $z \in\left(-\infty, \varepsilon_{\min }(k)\right)$ функция $\Delta_{\gamma \mu}^{*}(k ; z)$ представляется в виде

$$
\Delta_{\gamma \mu}^{*}(k ; z)=(\varepsilon(k)-z) \Delta_{\gamma \mu}(k ; z),
$$

поэтому нули функций $\Delta_{\gamma \mu}^{*}(k ; \cdot)$ и $\Delta_{\gamma \mu}(k ; \cdot)$ совпадают на $\left(-\infty, \varepsilon_{\min }(k)\right)$. Из того факта, что $\Delta_{\gamma \mu}(k ; \cdot)$ монотонно убывает на интервале $\left(-\infty, \varepsilon_{\min }(k)\right)$, следует, что функция $\Delta_{\gamma \mu}^{*}(k ; \cdot)$ имеет не более одного нуля на этом интервале.

Связь между собственными значениями самосопряженного оператора $H_{\gamma \mu}(k)$ и нулями функции $\Delta_{\gamma \mu}^{*}(k ; z)$ устанавливается в следующей лемме. 
Лемма 3. Для любъх $\gamma, \mu \in[0,+\infty)$ число $z \in \mathbb{C} \backslash\left[\varepsilon_{\min }(k), \varepsilon_{\max }(k)\right]$ является собственным значением оператора $H_{\gamma \mu}(k), k \in \mathbb{T}^{d}$, тогда и только тогда, когда $\Delta_{\gamma \mu}^{*}(k ; z)=0$.

ДоказАтельство. Необходимость. Пусть число $z \in \mathbb{C} \backslash \sigma_{\mathrm{ess}}\left(H_{\gamma \mu}(k)\right)$ есть собственное значение оператора $H_{\gamma \mu}(k), k \in \mathbb{T}^{d}$, и пусть $f=\left(f_{0}, f_{1}\right) \in \mathcal{H}$ - соответствующая собственная вектор-функция. Тогда $f_{0}$ и $f_{1}$ удовлетворяют следующей системе уравнений:

$$
\begin{aligned}
(\varepsilon(k)-z) f_{0} & =-\gamma\left(f_{1}, \alpha_{0}\right), \\
\left(\varepsilon_{k}(q)-z\right) f_{1}(q) & =\mu \alpha_{0}\left(f_{1}, \alpha_{0}\right)-\gamma \alpha_{0} f_{0} .
\end{aligned}
$$

В силу равенства $\sigma_{\text {ess }}\left(H_{\gamma \mu}(k)\right)=\left[\varepsilon_{\min }(k), \varepsilon_{\max }(k)\right]$ для любых $z \in \mathbb{C} \backslash\left[\varepsilon_{\min }(k), \varepsilon_{\max }(k)\right]$ и $q \in \mathbb{T}^{d}$ имеет место неравенство

$$
\varepsilon_{k}(q)-z \neq 0, \quad k \in \mathbb{T}^{d}
$$

Из второго уравнения системы (6) для $f_{1}$ имеем

$$
f_{1}(q)=\frac{\mu \alpha_{0} c-\gamma \alpha_{0} f_{0}}{\varepsilon_{k}(q)-z}
$$

где $c=\left(f_{1}, \alpha_{0}\right)$. Подставляя выражение $(7)$ для $f_{1}(\cdot)$ в первое равенство $(6)$, получим, что система уравнений (6) имеет ненулевое решение тогда и только тогда, когда система линейных уравнений

$$
\begin{gathered}
(\varepsilon(k)-z) f_{0}+\gamma c=0, \\
\gamma f_{0} a(k, z)+c(1-\mu a(k, z))=0
\end{gathered}
$$

имеет ненулевое решение, т.е. когда определитель $\Delta_{\gamma \mu}^{*}(k ; z)$ этой системы равен нулю, т.е. $\Delta_{\gamma \mu}^{*}(k ; z)=0$. Следовательно, собственная функция имеет вид

$$
f=\left(f_{0}, f_{1}\right), \quad f_{0}=\frac{-\gamma c}{\varepsilon(k)-z}, \quad f_{1}(q)=\frac{\mu \alpha_{0} c-\gamma \alpha_{0} f_{0}}{\varepsilon_{k}(q)-z} .
$$

Достаточность. Пусть для некоторых $k \in \mathbb{T}^{d}$ и $z \in \mathbb{C} \backslash\left[\varepsilon_{\min }(k), \varepsilon_{\max }(k)\right]$ выполняется равенство $\Delta_{\gamma \mu}^{*}(k ; z)=0$. Тогда вектор $f=\left(f_{0}, f_{1}\right)$ вида $(9)$ удовлетворяет системе уравнений $(6)$, т.е. число $z \in \mathbb{C} \backslash\left[\varepsilon_{\min }(k), \varepsilon_{\max }(k)\right]$ является собственным значением оператора $H_{\gamma \mu}(k), k \in \mathbb{T}^{d}$.

ЗАмЕчАниЕ 3 . Если $n$ координат вектора $k \in \mathbb{T}^{d}$ равны числу $\pi$, то $\mathcal{E}_{k}(\cdot)-\varepsilon_{\min }(k)$ можно рассматривать как функцию, определенную на $\mathbb{T}^{d-n}$ и имеющую невырожденный минимум в $\mathbb{T}^{d-n}$. Поэтому в следующей лемме без ограничения общности предположим, что $k \in \Pi_{0, \pi}$.

Лемма 4. А. Пусть $d \geqslant 3$. Тогда для любого $k \in \Pi_{0, \pi}$ функиия

$$
a\left(k, \varepsilon_{\min }(k)\right)=\frac{1}{(2 \pi)^{d}} \int_{\mathbb{T}^{d}} \frac{d q}{\varepsilon_{k}(q)-\varepsilon_{\min }(k)}
$$

является аналитической в $\Pi_{0, \pi}$. 
Б. Пусть $d \geqslant 1, k \in \Pi_{0, \pi} u z<\varepsilon_{\min }(k)$. Тогда функиия $a(k, z)$ представляется в виде

$$
\begin{aligned}
a(k, z)= & -\frac{\Phi_{0}(k)}{2}\left(z-\varepsilon_{\min }(k)\right)^{m} \ln \left(\varepsilon_{\min }(k)-z\right)+ \\
& +\left(z-\varepsilon_{\min }(k)\right)^{m+1} \ln \left(\varepsilon_{\min }(k)-z\right) \Phi_{11}(k, z)+\nu(k)+\Phi_{2}(k, z)
\end{aligned}
$$

$n p u d=2 m+2 u$

$$
\begin{aligned}
a(k, z)= & \frac{\pi \Phi_{0}(k)}{2} \frac{\left(z-\varepsilon_{\min }(k)\right)^{m}}{\sqrt{\varepsilon_{\min }(k)-z}}+ \\
& \quad+\left(\varepsilon_{\min }(k)-z\right)^{m+1 / 2} \Phi_{12}(k, z)+\nu(k)+\Phi_{2}(k, z)
\end{aligned}
$$

npu $d=2 m+1$. Здесъ

$$
\begin{aligned}
\Phi_{0}(k) & =\frac{c}{\sqrt{\cos \left(k_{1} / 2\right) \ldots \cos \left(k_{d} / 2\right)}}, \quad c=\text { const }, \\
\Phi_{12}(k, z) & =\sum_{l=0}^{\infty} b_{l}(k)\left(\varepsilon_{\min }(k)-z\right)^{l / 2}
\end{aligned}
$$

u $\Phi_{11}(k, \cdot)$ и $\Phi_{2}(k, \cdot), k \in \Pi_{0, \pi}$, - аналитические функиии в некоторой $\delta$-окрестности $V_{\delta}\left(\varepsilon_{\min }(k)\right)$ точки $z=\varepsilon_{\min }(k), \Phi_{2}\left(k, \varepsilon_{\min }(k)\right)=0, a b_{l}(k), l=0,1,2 \ldots,-$ некоторые действительные числа (см. [5]).

ДоказАтельство. А. Легко видеть, что для любого $k \in \Pi_{0, \pi}$ точка $q_{0}=0 \in \Pi_{0, \pi}$ является единственной невырожденной точкой минимума функции $\mathcal{E}_{k}(q)$. Поэтому в силу леммы Морса с параметром существует взаимно однозначное регулярное отображение $q=\phi_{k}(x)$ некоторого шара $W_{\beta}(0)$ радиуса $\beta>0$ с центром в начале координат на окрестность $U_{\delta}(0) \in \Pi_{0, \pi}$ точки $q_{0}=0$ такое, что

$$
\varepsilon_{k}(q)-z=\varepsilon_{\min }(k)-z+\sum_{j=1}^{d} x_{j}^{2} .
$$

Функцию $a(k, z)$ можно представить, как сумму следующих двух функций:

$$
a(k, z)=\int_{U_{\delta}(0)} \frac{d \eta}{\mathcal{E}_{k}(q)-z}+\int_{\mathbb{T}^{d} \backslash U_{\delta}(0)} \frac{d \eta}{\mathcal{E}_{k}(q)-z}=G_{1}(k, z)+G_{2}(k, z),
$$

где $G_{2}(\cdot ; z), z<\varepsilon_{\min }(k)$, и $G_{2}(k, \cdot), k \in \Pi_{0, \pi}$, - аналитические функции в $\Pi_{0, \pi}$ и на $\left(-\infty, \varepsilon_{\min }(k)\right)$ соответственно. Докажем, что функция $G_{1}\left(k, \varepsilon_{\min }(k)\right)$ аналитична в $\Pi_{0, \pi}$.

В первом интеграле равенства (10), делая замену переменных $q=\phi_{k}(x)$, где

$$
\phi_{k}: W_{\beta}(0) \rightarrow U_{\delta}(0), \quad q_{j} \mapsto \arccos \left(1-\frac{x_{j}^{2}}{2 \cos \left(k_{j} / 2\right)}\right),
$$

получим

$$
\begin{aligned}
G_{1}(k, z)= & \int_{W_{\beta}(0)} \frac{1}{\varepsilon_{\min }(k)-z+\sum_{j=1}^{d} x_{j}^{2}} \times \\
& \times \frac{2 d x_{1}}{\sqrt{2 \cos \left(k_{1} / 2\right)} \sqrt{2-x_{1}^{2} / 2 \cos \left(k_{1} / 2\right)}} \cdots \frac{2 d x_{d}}{\sqrt{2 \cos \left(k_{d} / 2\right)} \sqrt{2-x_{d}^{2} / 2 \cos \left(k_{d} / 2\right)}} .
\end{aligned}
$$


Переходя к сферическим координатам:

$$
\begin{aligned}
& x_{1}=r \cos \psi_{1} \cos \psi_{2} \ldots \cos \psi_{d-2} \cos \psi_{d-1}, \\
& x_{2}=r \cos \psi_{1} \cos \psi_{2} \ldots \cos \psi_{d-2} \sin \psi_{d-1}, \\
& x_{3}=r \cos \psi_{1} \cos \psi_{2} \ldots \sin \psi_{d-2}, \\
& \ldots \ldots \ldots \ldots \ldots \ldots \ldots, \ldots \ldots \ldots, \quad-\frac{\pi}{2} \leqslant \psi_{d} \leqslant \frac{\pi}{2}, \quad 0 \leqslant \psi_{d-1} \leqslant 2 \pi, \\
& x_{d}=r \sin \psi_{1}, \\
& 0 \leqslant r \leqslant \beta, \quad-\frac{\pi}{2} \leqslant \psi_{1} \leqslant \frac{\pi}{2}, \quad \ldots,
\end{aligned}
$$

получим

$$
\begin{aligned}
G_{1}(k, z)= & \frac{1}{\sqrt{\cos \left(k_{1} / 2\right) \ldots \cos \left(k_{d} / 2\right)}} \times \\
& \times \int_{0}^{\beta} \int_{-\pi / 2}^{\pi / 2} \int_{-\pi / 2}^{\pi / 2} \ldots \int_{0}^{2 \pi} \frac{r^{d-1} \omega(\psi)}{\varepsilon_{\min }(k)-z+r^{2}} \times \\
& \times \frac{d r d \psi_{d-1} \ldots d \psi_{1}}{\sqrt{1-r^{2} \omega_{1}^{2}(\psi) / 2 \cos \left(k_{1} / 2\right)} \ldots \sqrt{1-r^{2} \omega_{d}^{2}(\psi) / 2 \cos \left(k_{d} / 2\right)}}
\end{aligned}
$$

где

$$
\begin{aligned}
\omega(\psi) & =\omega\left(\psi_{1}, \ldots, \psi_{d-1}\right)=\cos ^{d-2} \psi_{1} \cos ^{d-3} \psi_{2} \ldots \cos \psi_{d-2} \\
\omega_{1}(\psi) & =\omega\left(\psi_{1}, \ldots, \psi_{d-1}\right)=\cos \psi_{1} \cos \psi_{2} \ldots \cos \psi_{d-1}
\end{aligned}
$$

и

$$
\omega_{j}(\psi)=\omega\left(\psi_{1}, \ldots, \psi_{d-1}\right)=\cos \psi_{1} \cos \psi_{2} \ldots \sin \psi_{d-j+1}, \quad j=2, \ldots, d .
$$

Поскольку при $d \geqslant 3$ существует предел

$$
\begin{aligned}
G_{1}\left(k, \varepsilon_{\min }(k)\right)= & \lim _{z \rightarrow \varepsilon_{\min }(k)} G_{1}(k, z)=\frac{1}{\sqrt{\cos \left(k_{1} / 2\right) \ldots \cos \left(k_{d} / 2\right)}} \times \\
& \times \int_{0}^{\beta} r^{d-3} d r \int_{-\pi / 2}^{\pi / 2} \int_{-\pi / 2}^{\pi / 2} \ldots \int_{0}^{2 \pi} \omega(\psi) \times \\
& \times \frac{d r d \psi_{d-1} \ldots d \psi_{1}}{\sqrt{1-r^{2} \omega_{1}^{2}(\psi) / 2 \cos \left(k_{1} / 2\right)} \ldots \sqrt{1-r^{2} \omega_{d}^{2}(\psi) / 2 \cos \left(k_{d} / 2\right)}}
\end{aligned}
$$

и $\beta>0$ достаточно мало, то функция $G_{1}\left(k, \varepsilon_{\min }(k)\right)$ аналитична в $\Pi_{0, \pi}$, и, следовательно, в силу (10) функция $a\left(k, \varepsilon_{\min }(k)\right)$ аналитична в $\Pi_{0, \pi}$.

Утверждение Б доказывается аналогично лемме 4 в работе [5].

ДокАЗАТЕЛЬСтво теОремы 1. Пусть $z<\varepsilon_{\min }(k)$. В силу лемм 2 и 4 при фиксированных $\gamma>0, \mu \geqslant 0$ имеют место равенства

$$
\lim _{z \rightarrow \varepsilon_{\min }(k)-0} \Delta_{\gamma \mu}(k ; z)=-\infty, \quad \lim _{z \rightarrow-\infty} \Delta_{\gamma \mu}(k ; z)=1 .
$$

Поскольку непрерывная функция $\Delta_{\gamma \mu}(k ; \cdot)$ монотонно убывает и меняет на интервале $\left(-\infty, \varepsilon_{\min }(k)\right)$ знак, то уравнение $\Delta_{\gamma \mu}(k ; z)=0$ имеет единственное решение 
$z=E_{\gamma \mu}(k)$ на этом интервале. Следовательно, согласно леммам 2 и 3 оператор $H_{\gamma \mu}(k)$ имеет единственное собственное значение $E_{\gamma \mu}(k)<\varepsilon_{\min }(k)$.

Вещественная аналитичность функции $E_{\gamma \mu}(k)$ на $\mathbb{T}^{d}$ следует из того факта, что $E_{\gamma \mu}(k)$ является единственным решением уравнения $\Delta_{\gamma \mu}(k ; z)=0$, а также из того, что функция $\Delta_{\gamma \mu}(k ; \cdot)$ вещественно-аналитична в $\mathbb{C} \backslash\left[\varepsilon_{\min }(k), \varepsilon_{\max }(k)\right]$ и функция $\Delta_{\gamma \mu}(\cdot ; z), z \in \mathbb{C} \backslash\left[\varepsilon_{\min }(k), \varepsilon_{\max }(k)\right]$ вещественно-аналитична на торе $\mathbb{T}^{d}$. Так как $\Delta_{\gamma \mu}(\cdot ; z)$ - аналитическая и четная функция на $\mathbb{T}^{d}$, то $E_{\gamma \mu}(\cdot)$ является четной вещественно-аналитической функцией.

Заметим, что число $E_{\gamma \mu}(k) \in \mathbb{C} \backslash\left[\varepsilon_{\min }(k), \varepsilon_{\max }(k)\right]$ есть собственное значение оператора $H_{\gamma \mu}(k), k \in \mathbb{T}^{d}$, тогда и только тогда, когда система уравнений

$$
\begin{aligned}
\left(\varepsilon(k)-E_{\gamma \mu}(k)\right) f_{0} & =-\gamma\left(f_{1}, \alpha_{0}\right), \\
\left(\varepsilon_{k}(q)-E_{\gamma \mu}(k)\right) f_{1}(q) & =\mu \alpha_{0}\left(f_{1}, \alpha_{0}\right)-\gamma \alpha_{0} f_{0}
\end{aligned}
$$

имеет нетривиальное решение $f_{k}=\left(f_{0, k}, f_{1, k}\right) \in \mathcal{H}$, где

$$
f_{0, k}=\frac{-\gamma c}{\varepsilon(k)-E_{\gamma \mu}(k)}, \quad f_{1, k}(q)=\left(\frac{\gamma^{2}}{\varepsilon(k)-E_{\gamma \mu}(k)}+\mu\right) \frac{c}{\varepsilon_{k}(q)-E_{\gamma \mu}(k)},
$$

$c \neq 0$ - нормирующий множитель. Из выражения (13) для $f_{0, k}$ и $f_{1, k}$ и из аналитичности функции $E_{\gamma \mu}(\cdot)$ на $\mathbb{T}^{d}$ можно заключить, что отображение $f: \mathbb{T}^{d} \rightarrow \mathcal{H}$, $k \rightarrow f_{k} \in \mathcal{H}$ является аналитическим отображением на $\mathbb{T}^{d}$. Аналитичность отображения $f: k \rightarrow f_{k} \in \mathcal{H}$ следует из аналитичности компонент $f_{0, k}$ и $f_{1, k}$ вектора $f_{k}$.

ДокАЗАТЕЛЬСТво теОремы 2. Пусть $k \in \widetilde{\Pi}_{0, \pi}$ и $z<\varepsilon_{\min }(k)$. В силу лемм 2 и 4 при фиксированных $\gamma>0, \mu \geqslant 0$ имеют место равенства

$$
\lim _{z \rightarrow \varepsilon_{\min }(k)-0} \Delta_{\gamma \mu}(k ; z)=-\infty, \quad \lim _{z \rightarrow-\infty} \Delta_{\gamma \mu}(k ; z)=1 .
$$

Поскольку непрерывная функция $\Delta_{\gamma \mu}(k ; \cdot)$ монотонно убывает и меняет на интервале $\left(-\infty, \varepsilon_{\min }(k)\right)$ знак, то из утверждения В леммы 2 следует, что уравнение $\Delta_{\gamma \mu}^{*}(k ; z)=0$ имеет единственное решение $z=E_{\gamma \mu}(k)$ на этом интервале. Следовательно, согласно лемме 3 оператор $H_{\gamma \mu}(k)$ имеет единственное собственное значение $E_{\gamma \mu}(k)<\varepsilon_{\min }(k)$.

ДокАЗАТЕЛЬСТво ТЕОРЕМЫ 3. А. Пусть вектор $k \neq \vec{\pi}$ такой, что каждая из его координат равна либо нулю, либо $\pi$. Предположим, что число $z=\varepsilon_{\min }(k)$ является решением уравнения

$$
H_{\gamma \mu}(k) f=\varepsilon_{\min }(k) f, \quad f=\left(\begin{array}{l}
f_{0} \\
f_{1}
\end{array}\right), \quad f_{0} \in \mathbb{C}, \quad f \in L^{1}\left(\mathbb{T}^{d}\right) \text { или } L^{2}\left(\mathbb{T}^{d}\right) .
$$

Тогда система уравнений (6) принимает вид

$$
\begin{aligned}
\int_{\mathbb{T}^{d}} f_{1}(q) & =0, \\
{\left[\varepsilon_{k}(q)-\varepsilon_{\min }(k)\right] f_{1}(q) } & =-\gamma \alpha_{0} f_{0},
\end{aligned}
$$

где $\alpha_{0} \neq 0$. Учитывая первое равенство, из второго уравнения получим, что

$$
f_{1}(q)=-\frac{\gamma \alpha_{0} f_{0}}{\varepsilon_{k}(q)-\varepsilon_{\min }(k)}
$$


где $\varepsilon_{k}(q)-\varepsilon_{\min }(k) \geqslant 0$ - непрерывная функция, не равная тождественно нулю. Но эта функция удовлетворяет первому уравнению системы (14) только при $f_{0}=0$. Следовательно, $f_{1}=0$. Это означает, что число $z=\varepsilon_{\min }(k)$ не является ни собственным значением, ни виртуальным уровнем оператора $H_{\gamma \mu}(k)$.

Б. Если $k=\vec{\pi}$, то система уравнений (6) имеет вид

$$
\begin{gathered}
\gamma\left(f_{1}, \alpha_{0}\right)=0, \\
\gamma \alpha_{0} f_{0}=0 .
\end{gathered}
$$

В силу того что $\alpha_{0} \neq 0$, отсюда следует утверждение Б теоремы.

ДоказАтЕЛЬство теоремы 4. Утверждение А теоремы очевидно. Утверждение Б доказывается аналогично теореме 1.

Докажем утверждение В. Так как разложение функции $\varepsilon_{k}(q)-\varepsilon_{\min }(k)$ в окрестности невырожденного минимума $q=0$ начинается с $q^{2}=q_{1}^{2}+\cdots+q_{d}^{2}$, несобственные интегралы

$$
\int_{\mathbb{T}^{d}} \frac{d q}{\left[\varepsilon_{k}(q)-\varepsilon_{\min }(k)\right]^{n}}, \quad \int_{\mathbb{T}^{d}} \frac{d q}{q^{2 n}}, \quad n=1,2, \ldots,
$$

сходятся или расходятся одновременно (см. доказательство леммы 4). Отсюда следует, что $f_{1, k} \in L_{e}^{1}\left(\mathbb{T}^{d}\right) \backslash L_{e}^{2}\left(\mathbb{T}^{d}\right)$ при $k \in\left[\Pi_{d-3, \pi} \cup \Pi_{d-4, \pi}\right] \cap \widetilde{\Pi}_{0, \pi}^{\perp}$, и $f_{1, k} \in L_{e}^{2}\left(\mathbb{T}^{d}\right)$ при $k \in \Pi_{\pi}^{(3)} \cap \widetilde{\Pi}_{0, \pi}^{\perp}$. Второе утверждение следует из определения виртуального уровня и множества $G_{\gamma \mu}^{0}$.

Г. Из определения $G_{\gamma \mu}^{>}$и $G_{\gamma \mu}^{0}$ следует, что при $k \in G_{\gamma \mu}^{>} \cup G_{\gamma \mu}^{0}$ имеет место неравенство $\Delta_{\gamma \mu}\left(k, \varepsilon_{\min }(k)\right) \geqslant 0$. Далее, имеет место представление $\Delta_{\gamma \mu}^{*}(k, z)=(\varepsilon(k)-$ $z) \Delta_{\gamma \mu}(k, z)$, а функция $\Delta_{\gamma \mu}(k, z)$ монотонно убывает на интервале $\left(-\infty, \varepsilon_{\min }(k)\right)$. Поэтому в силу леммы 2 уравнение $\Delta_{\gamma \mu}^{*}(k, z)=0$ не имеет решения на этом интервале и согласно лемме 3 оператор $H_{\gamma \mu}(k)$ не имеет собственного значения на интервале $\left(-\infty, \varepsilon_{\min }(k)\right)$.

Благодарности. Авторы приносят глубокую благодарность Р. А. Минлосу за внимание и обсуждения, а также рецензенту за полезные замечания.

\section{Список литературы}

[1] A. K. Motovilov, W. Sandhas, V. B. Belyaev, J. Math. Phys., 42:6 (2001), 2490-2506.

[2] S. Albeverio, S. N. Lakaev, T. H. Rasulov, J. Stat. Phys., 127:2 (2007), 191-220.

[3] S. Albeverio, S. N. Lakaev, K. A. Makarov, Z. I. Muminov, Commun. Math. Phys., 262:1 (2006), 91-115, arXiv: math-ph/0501013.

[4] М. Рид, Б. Саймон, Методы современной математической физики, т. 4: Анализ операторов, Мир, М., 1982.

[5] С. Н. Лакаев, ТМФ, 91:1 (1992), 51-65. 